\title{
Périodicité du phytoplancton dans un réservoir de moyenne profondeur : le lac de Pareloup (Massif central, France), un exemple de succession cyclique
}

\author{
R. Le Cohu ${ }^{1}$ \\ N. Comoy! \\ J. Guitard ${ }^{1}$ \\ J. Brabet 1
}

Mots clés : Réservoir, phytoplancton, biomasse, variations saisonnières, succession cyclique.

L'étude de la dynamique saisonnière du phytoplancton dans le lac de Pareloup a été réalisée dans le cadre plus général de la mise en ouvre d'un modèle de fonctionnement de ce réservoir en vue de sa gestion. La biomasse (poids frais $\mu \mathrm{g} .1^{-1}$ et chlorophylle a) et la densité cellulaire ont été suivies de mars à novembre pendant deux années. Le maximum annuel de phytoplancton (biomasse et densité cellulaire) se situe au printemps ; il est dominé par les diatomées dont le déclin est initié par la carence en silice du milieu et, sans doute, par des concentrations cellulaires en chlorophylle a minimales. Une phase claire résulte de la sédimentation des diatomées et se prolonge pendant tout l'été. Cette période estivale se caractérise par une forte production primaire et une faible biomasse, biomasse régulée par la prédation. Avec le brassage automnal, de petites espèces connaissent une croissance rapide qui s'exprime seulement en densité cellulaire ; la faible augmentation des valeurs de biomasse par rapport à celles de l'été est due essentiellement à la présence de Gonyostomum semen et au léger renouveau des diatomécs. Les différentes phases du développement du phytoplancton sont analysées et confrontées au modèle P.E.G. proposé par Sommer et al. (1986). La mise en place de la stratification thermique est l'élément fondamental dans le fonctionnement du réservoir de Pareloup : chaque année, elle détermine le départ d'une succession phytoplanctonique de type cyclique.

The periodicity of phytoplankton in a shallow reservoir : the Pareloup Lake (Central Massif, France), an example of cyclic succession

Keywords : Reservoir, phytoplankton, biomass, seasonal periodicity, cyclic succession.

A study of the seasonal dynamics of the phytoplankton in the Pareloup Lake has been performed in the general context of developing a functional model for the management of the reservoir. Biomass (wet weight $\mu \mathrm{g} . \mathrm{l}^{-1}$ and chlorophyll a) and cell density have been monitored from March to November over two years. The annual maximum for phytoplankton (biomass and cell density) occurs in spring ; it is dominated by diatoms and their decline starts with a lack of silica in the environment and also with minimum cellular concentrations of chlorophyll a. A clear phase results from the sedimentation of the diatoms and lasts through the summer. In autumn, there is a marked increase in primary production but a low biomass, the latter being regulated by predation. With the autumn mixing, small species experience a rapid growth which is detected only in terms of cell density; the poor increase in biomass values compared with those of the summer is due chiefly to the presence of Gonyostomum semen and the slight revival of diatoms. The different phases of phytoplankton development are analysed and compared with the P.E.G. model proposed by Sommer et al. (1986). The establishment of thermal stratification is the dominant element in the functioning of the Pareloup reservoir, each year, it determines the start of a cyclic phytoplankton succession.

1. Laboratoire d'Hydrobiologic, U.R.A. 695 du C.N.R.S., Université Paul Sabatier, 118, route de Narbonne, F-31062 Toulouse Cedex. 


\section{Introduction}

Le développement des populations algales est régi par des facteurs chimiques, biologiques (prédation, parasitisme) et physiques (Reynolds 1984, Sommer et al. 1986 et Sommer 1989a). Ces différents paramètres jouent un rôle plus ou moins décisif suivant les périodes de l'année, ce qui entraine des fluctuations dans la composition et l'abondance de la flore algale ; la modélisation du fonctionnement d'un lac en vue de sa gestion (Thébault \& Salençon, sous presse) nécessite donc, entre autres, la connaissance de la dynamique saisonnière du phytoplancton : celle-ci a été étudiée dans le lac de Pareloup parallèlement à d'autres approches (Rhodes \& Le Cohu 1986, Capblancq et al. 1988, Lavandier 1990, Labroue 1990, Le Jolivet 1988).

Le lac de Pareloup constitue l'une des plus vastes retenues hydroélectriques de France : il est situé dans le Sud-Ouest du Massif central où, jusqu'à maintenant, ce sont surtout des lacs naturels qui ont fait l'objet d'études (Devaux 1973 et 1977, Amblard 1988, Amblard \& Bourdier 1990).

Dans ce travail, sont présentées et analysées les variations saisonnières de la biomasse totale (poids frais et chlorophylle), des différents groupes d'algues et de quelques espèces; dans la conclusion, les résultats obtenus sont confrontés au modèle P.E.G. proposé par Sommer et al. (1986).

\section{Méthodes et principales caractéristiques du site étudié}

Des prélèvements, hebdomadaires le plus souvent, ont été effectués de mars à novembre pendant 2 années (1986 et 1987) en un point où la profondeur atteignait $32 \mathrm{~m}$. Pendant la période d'isothermie, ils ont porté sur les espaces 0-8 m, 8-16 m et $16-25 \mathrm{~m}$; pendant la période de stratification, ils ont été effectués tous les mètres. A partir de 1987, la fixation des algues au formol a été abandonnée au profit de la fixation au glutaraldéhyde neutralisé par le cacodylate de sodium, ce qui a permis, une meilleure conservation des flagellés en particulier des cellules de Gonyostomum semen (Ehr.) Diesing et de les compter. Les comptages ont été effectués au microscope inversé ( $L$ und et al. 1958). La densité cellulaire est donnée en nombre de cellules par litre. La biomasse est exprimée d'une part en poids frais $(\mu \mathrm{g}$. $\mathrm{1}^{-1}$ ) déterminé après estimation du volume des cellules et d'autre part en chlorophylle a. Dans le cas de Gomphosphaeria naegeliana (Unger) Lemm., le biovolume a été évalué à partir des colonies, la densité cellulaire a été obtenue par calcul à partir du nombre moyen de cellules par colonie.

La transparence de l'eau a été déterminée à l'aide du disque de Secchi. L'azote nitrique et la silice ont été dosés au technicon « analyzer », les différentes formes de phosphore suivant la méthode de Motomizu et al. (1983). Pour la chlorophylle, la technique d'extraction utilisant l'éthanol bouillant (Marker et al. 1980) a été adoptée.

Le réservoir de Pareloup est de type monomictique chaud avec une stratification très stable de la fin juin à mi-septembre. Les principales caractéristiques de la retenue sont consignées dans le tableau 1. Les figures 1 et 2 décrivent les cycles annuels de l'azote nitrique (N-NO 3$)$, du phosphore réactif soluble (P.R.S.), du phosphore total (Pt), de la silice $\left(\mathrm{SiO}_{2}\right)$, du rapport $\mathrm{Si} / \mathrm{Pt}$ et du rapport $\mathrm{N}-\mathrm{NO}_{3} / \mathrm{Pt}$.

Tableau $\downarrow$. Principales caractéristiques de la retenue d'eau de Pareloup.

\begin{tabular}{ll}
\hline Altitude du plan d'eau & $850 \mathrm{~m}$ \\
Superficie & $13,5 \mathrm{~km}^{2}$ \\
Volume utile & $168.10^{6} \mathrm{~m}^{3}$ \\
Longueur & $7,7 \mathrm{~km}$ \\
Largeur moyenne & $1,7 \mathrm{~km}$ \\
Profondeur maximum & $35 \mathrm{~m}$ \\
Profondeur moyenne & $12,5 \mathrm{~m}$ \\
Développement de la ligne de côte & $790-804 \mathrm{~m}$ \\
Superficie du bassin versant & $122 \mathrm{~km}^{2}$ \\
Superficie totale du bassin drainé & $385 \mathrm{~km}^{2}$ \\
Débit moyen turbiné & $7,3 \mathrm{~m}^{3} . \mathrm{s}^{-1}$ \\
Débit réservé & $1001 . \mathrm{s}^{-1}$ \\
\hline
\end{tabular}

\section{Résultats}

\subsection{Evolution saisonnière du phytoplancton global}

3.1.1. Variation de la biomasse et de la densité cellulaire (fig. 3)

Les variations saisonnières de la biomasse phytoplanctonique se reproduisent en 1986 et 1987 suivant le même modèle. Le maximum annuel s'établit chaque année rigoureusement aux mêmes dates, que les résultats soient exprimés en poids frais ou en densité cellulaire. Comme dans beaucoup de lacs de profondeur moyenne (Hutchinson 1967, Reynolds 1984, Sommer et al. 1986), ce pic printanier est dû essentiellement aux diatomées qui peuvent 

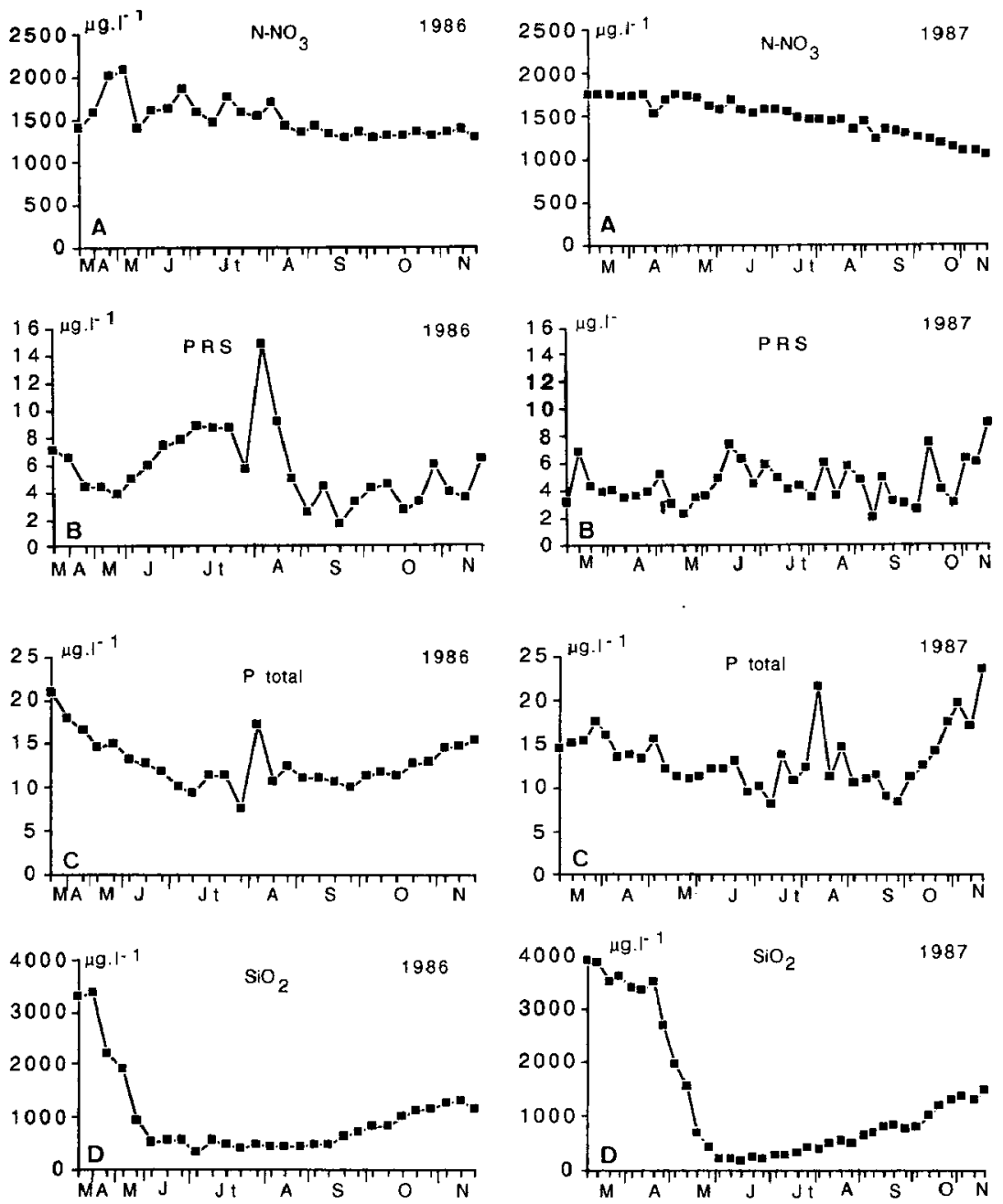

Fig. 1. Cycle annuel (zone euphotique) : A - de l'azote nitrique ( $\mathrm{N}-\mathrm{NO}_{3}$ ), B - du phosphore réactif soluble (PRS), C - du phosphore total (P total), D - de la silice $\left(\mathrm{SiO}_{2}\right)$. 

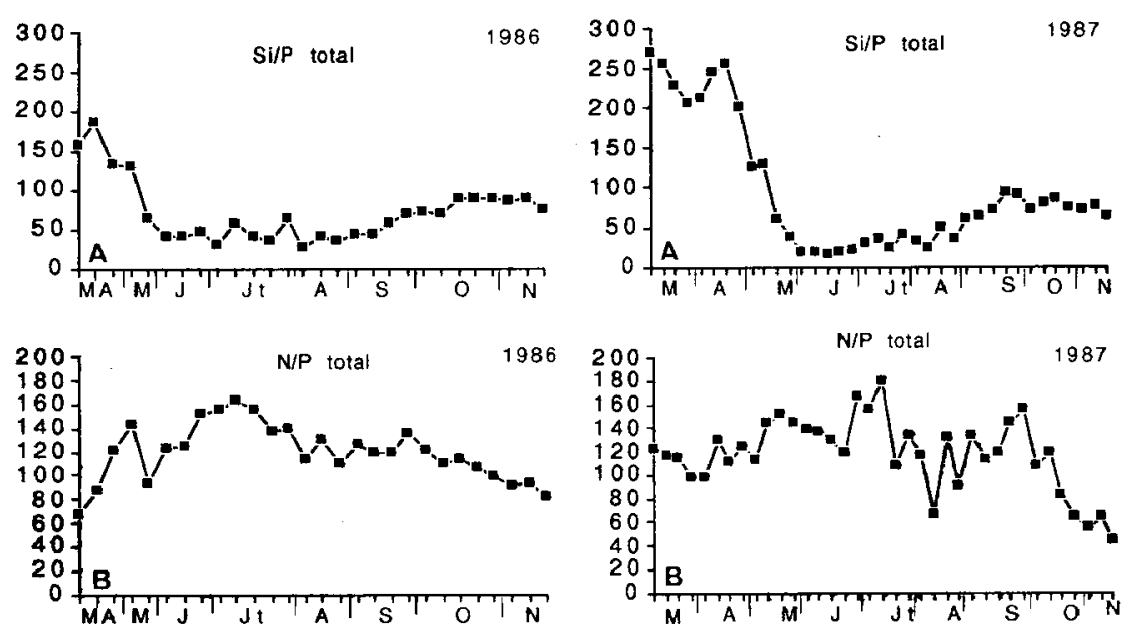

Fig. 2. Variations annuelies des rapports $\mathrm{Si} / \mathrm{P}$ total (A) et $\mathrm{N}-\mathrm{NO}_{3} / \mathrm{P}$ total (B).
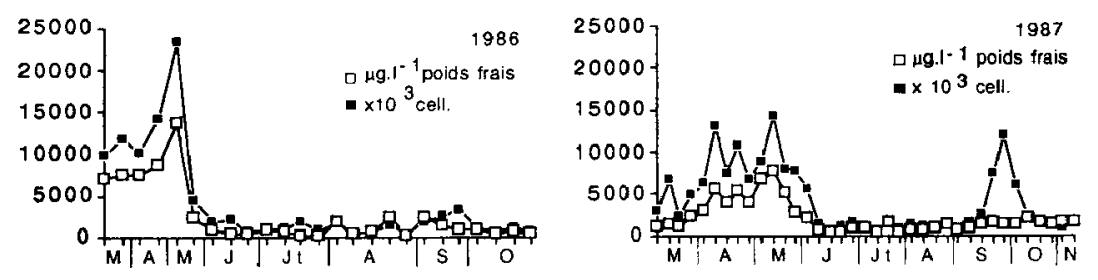

Fig. 3. Evolution annuelle de la biomasse du phytoplancton ( $\mu \mathrm{g} .1^{-1}$ de poids frais) et de la densité cellulaire (10 cell. $\left.\mathbf{I}^{-1}\right)$ dans la zone euphotique. 
représenter $96 \%$ de la biomasse phytoplanctonique totale en $1986^{*}$ et jusqu'à $99 \%$ en 1987 (fig. 6). Ce développement massif des diatomées provoque une diminution drastique de la silice (fig. 1 D), qui débute un mois avant le maximum printanier. Les concentrations en silice passent de $3,5 \mathrm{mg} . \mathrm{l}^{-1}$ à $0,31 \mathrm{mg} . \mathrm{l}^{-1}$ en 1986 et de $3.9 \mathrm{mg} .1^{-1}$ à $0,17 \mathrm{mg} . \mathrm{l}^{-1}$ en 1987. Dès la mi-mai, la biomasse phytoplanctonique décroit rapidement pour tomber à des valeurs très basses (fig. 3), ce qui se traduit par une clarification des eaux ; la transparence de l'eau mesurée au disque de Secchi passe de 2-3 $\mathrm{m}$ en mars à 6-7 $\mathrm{m}$ en juillet. Il faut observer que la chute de la biomasse ainsi que la majeure partie du processus de sédimentation sont achevés avant la mise en place de la stratification thermique estivale (données non publiées) ; pendant toute cette période se prolonge la phase des eaux claires, marquée seulement par quelques petits pies secondaires de biomasse dus à des poussées d'algues de différents groupes, diatomées exceptées (fig. 6). Au début de l'automne, lorsque la zone de brassage des eaux s'approfondit, on observe une augmentation de la densité cellulaire (fig. 3), surtout nette en 1987. Ce développernent algal est loin de se traduire par des valeurs de biomasse comparables à celles du printemps ; le peuplement automnal est constitué majoritairement d'espèces de très petite taille, ce qui contraste avec le peuplement vernal constitué surtout d'Asterionella formosa Hassal ; c'est ainsi que Merismopedia tenuissima Lemm. ne représente, malgré une forte densité cellulaire (jusqu'à $12.10^{6}$ cell. $1^{-}{ }^{\circ}$, le $29 / 9 / 87$ à $9 \mathrm{~m}$ ), qu'un faible pourcentage de la biomasse phytoplanctonique essentiellement formée de Gonyostomum semen et de diatomées. Le réservoir de Pareloup a un cycle de développement du phytoplancton assez proche de celui observé dans deux autres lacs du Massif central (Pavin et Tazenat) où les valeurs maximales de biomasse se situent à la fin de l'hiver et au printemps (Devaux 1977, Amblard \& Bourdier 1990).

\subsubsection{Evolution temporelle et spatiale de la chlo- rophylle a (fig. 4 et 5 ).}

Au cours de l'année, les concentrations en chlorophylle a (fig. 4) varient dans une proportion de 1 à 10 en 1986 et de 1 à 6 en 1987 ; les valeurs de la biomasse (en $\mu \mathrm{g} \cdot 1^{-1}$ poids frais) montrent des différences plus marquées, de 1 à 29 en 1986 et de 1 à 25 en 1987 . Pendant la période de floraison printanière, on observe un décalage très net entre les maximums de chlorophylle a et de biomasse phytoplanctonique (en $\mu \mathrm{g} .1^{-1}$ poids frais) ou de densité cellulaire (fig. 3) ; ce décalage de deux mois en 1986 et de trois semaines en 1987 reflete une diminution des concentrations cellulaires en chlorophylle a lorsque la densité cellulaire augmente ; en considérant la période pendant laquelle $A$. formosa représente
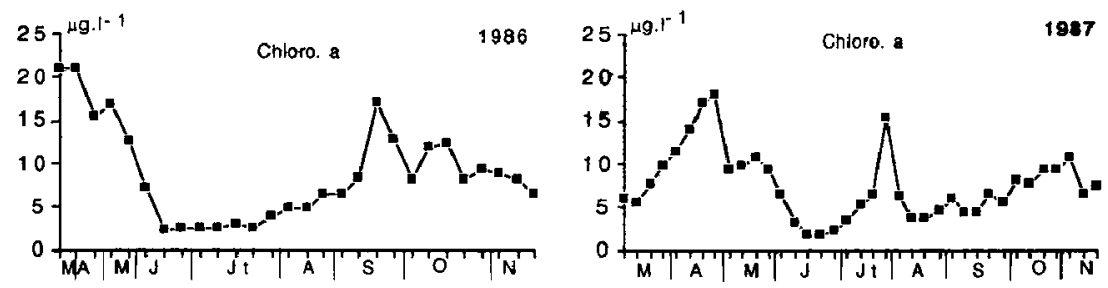

Fig. 4. Variations annuelles de la chlorophylle a dans la zone euphotique.

\footnotetext{
- N.B. En 1986, la représentation des différents groupes (en $\%$ ) au sein de la biomasse pendant la période de stratification et đurant l'automne est surévaluée du fait de la non-prise en compte de Gonyosiomum semen dans les comptages, c'est pourquoi nous n'en don. nons pas l'histogramme même si nous en faisons mention.
} 

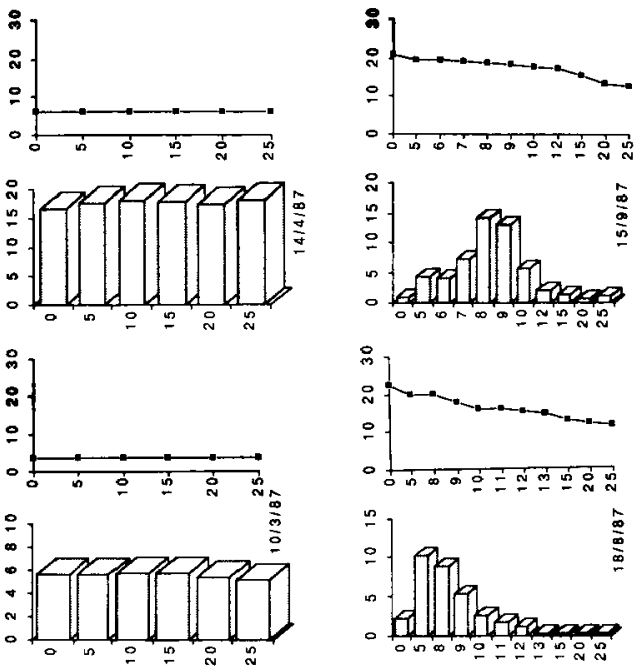

$\frac{\infty}{5}$
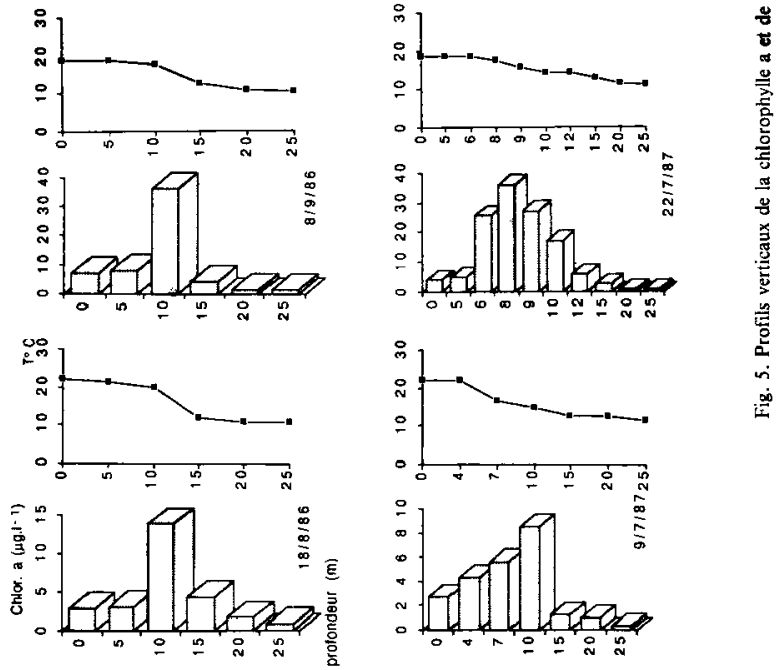


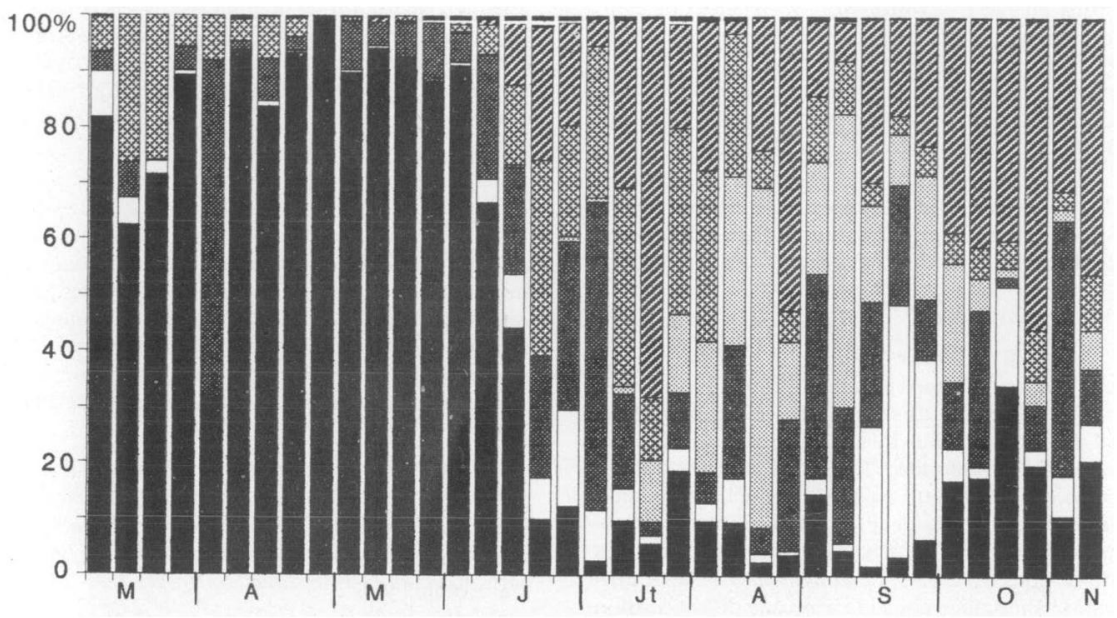

\section{E Nannoplancton Raphidophycées Pyrrhophycées Cyanophycées \\ Chlorophycées $\square$ Chrysophycées Diatomophycées}

Fïg. 6. Evolution de la structure du peuplement algal au cours de l'année 1987 dans la zone euphotique (To de biomasse).

plus de $80 \%$ de la biomasse, le contenu des cellules en chlorophylle a, calculé en divisant la concentration en chlorophylle par le nombre de cellules, varie entre $2 \mathrm{pg}$ et $0,8 \mathrm{pg}$ en 1986 et entre $2 \mathrm{pg}$ et $0,5 \mathrm{pg}$ en 1987. Ces valeurs confirment les données antérieures ( Jrad 1989) et sont proches des concentrations cellulaires publiées par Reynolds (1984) pour cette espèce. Dans le lac de Pareloup, les minimums cellulaires en chlorophylle a coincident avec les maximums de densité cellulaire ; ils précèdent donc de peu la baisse brutale du peuplement diatomique.

Pendant toute la période du développement vernal des diatomées, les profils verticaux de la chlorophylle a (fig. 5) sont relativement homogènes sur toute la colonne d'eau, ce qui signifie qu'une grande partie de la biomasse phytoplanctonique (plus de 60 $\%$ en biovolume) se trouve au dessous de la zone euphotique au moment des prélèvements. Le temps de séjour des diatomées dans la zone euphotique, est donc relativement bref ; en général, les algues tendent à compenser le faible éclairement moyen auquel elles sont soumises quotidiennement, par une augmentation du contenu des cellules en chlorophylle a (Falkowski 1984, Hoepffner 1984). La diminution des quotas cellulaires en chlorophylle a observée à Pareloup jointe à la diminution drastique des teneurs en $\mathrm{SiO}_{2}$ expliquent probablement le déclin brutal du peuplement diatomique avant l'établissement de la stratification estivale. Dès que celleci est installèe, la répartition verticale de la chlorophylle a montre des discontinuités très marquées (fig. 5) ; suivant les profondeurs, les concentrations en chlorophylle a peuvent varier de 1 à $7\left(5 \mu \mathrm{g} . \mathrm{l}^{-1}\right.$ $\rightarrow 35 \mu \mathrm{g} .1-1)$. Généralement les pics de chlorophylle a se placent entre 8 et $10 \mathrm{~m}$ de profondeur, juste au dessus et dans la partie supérieure du métalimnion. Les maximums de chlorophylle a en profondeur sont liés le plus souvent à de courtes périodes de 
croissance de Gonyostomum semen (Le Cohu et al. 1989) mais parfois de petits pics peuvent être assurés par un mélange d'autres espèces telles Dictyosphaerium pulchellum Wood, Gymnodinium sp., Gomphosphaeria naegeliana, Anabaena spiroides Klebh., Dinobryon bavaricum Imhof.

\subsection{Evolution de la structure du peuplement (fig. 6)} - Dynamique saisonnière de quelques genres ou espèces

\subsubsection{Diatomées}

Même si en 1986 les résultats sont biaisés du fait que Gonyostomum semen n'ait pas été pris en compte en raison de sa très mauvaise conservation (il n'est pas douteux que les pics de chlorophylle a observés en profondeur le $18 / 8 / 86$ et le $8 / 9 / 86$, fig. 5 , soient dus à cette espèce), les fluctuations dans la composition du peuplement phytoplanctonique présentent le même schéma général au cours des deux années d'étude ; l'établissement de la stratification thermique coincide avec une diversification des groupes (fig. 6). Le développement vernal des diatomées constitue, comme on l'a déjà signalé, le maximum annuel de biomasse ; ce maximum est dû essentiellement à la prolifération d'Asterionella formosa, espèce qui peut représenter jusqu'à $99 \%$ de la biomasse (fig. 6). Les données antérieures depuis 1981 (Lair, comm. pers., Jrad 1989) confirment la continuité et la prépondérance de cette espèce lors de la floraison printanière, continuité peu comparabìe à ce qui a pu être observé dans d'autres lacs (Lund 1964). D'autres espèces peuvent être associées à Asterionella formosa ; elles peuvent être abondantes mais elles ne présentent jamais une part importante de la biomasse ; ce sont par ordre décroissant d'importance : Aulacoseira ambigua (Grun.) Sim. (Syn. Melosira ambigua (Grun.) O. Müll.), Aulacoseira subarctica (O. Müll.) Haworth (Syn. : Melosira italica (Ehr.) Kütz subsp. subarcica O. Müll.), Cyclotella comta (Ehr.) Kütz et Stephanodiscus minutulus (Kütz) Cleve et Möller. Dans les lacs naturels du Massif central de type dimictique, Asterionella formosa n'apparaît jamais comme une espèce dominante, elle est remplacée par des espèces telles Synedra acus Kutz. ou Aulacoseira subarctica (Amblard 1988, Devaux 1973 et 1977). Dans le lac de Pareloup, les diatomées subsistent pendant l'été, avec toujours Asterionella formosa comme espece la plus fréquente, mais elles sont très minoritaires dans la constitution de la biomasse. Elles connaissent un léger regain après la destruction de la stratification thermique et une augmentation des concentrations en $\mathrm{SiO}_{2}$ (fig. 1 D) mais leur développement reste toujours largement inférieur à celui observé au printemps à Pareloup.

\subsubsection{Chrysophycées}

Au sein de la biomasse phytoplanctonique, les chrysophycées ne se manifestent jamais comme un élément prépondérant ; en valeur relative elles peuvent atteindre $40 \%$ de la biomasse à l'époque où celle-ci est faible ; c'est ce qui peut être observé au moment de la stratification estivale pendant une courte période en juillet 1986 et en septembre 1987 (fig. 6).

Dans le réservoir de Pareloup, trois genres sont présents : le genre Mallomonas avec $M$. akrokomos Ruttn. et $M$. caudata Iwanoff. ; le genre Dinobryon avec $D$. sertularia Ehr. et $D$. bavaricum Imhof et enfin le genre Ochromonas.

Le genre Mallomonas, avec notamment $M$. akrokomos, a été détecté dans $75 \%$ des prélèvements en 1986 et dans $86 \%$ en $1987 ; M$. caudata a une présence sporadique (une cellule de $M$. caudata équivaut à 10 cellules de $M$. akrokomos en biovolume). Le maximum annuel (fig. $7 \mathrm{~A}$ ) s'établit toujours en fin d'hiver-début de printemps et est dû essentiellement à $\boldsymbol{M}$. akrokomos; ce développement vernal peut être éphémère (1987) ou s'étaler jusqu'à la fin du printemps (1986). Il faut toutefois observer qu'en 1987 le pic de densité cellulaire est 3,5 fois plus élevé que l'année précédente. Les deux années, pendant toute la période de stratification, $M$. akrokomos est présent même si la densité cellulaire n'est jamais très élevée. Cette espéce peut s'adapter à des concentrations en azote ammoniacal très importantes ( 5 mg. $1^{-1}$ ) conjointement à des teneurs en azote nitrique de l'ordre de $10 \mathrm{mg} .1-1$ (Le Cohu 1973). Aussi bien en 1986 qu'en 1987, le genre Mallomonas ne constitue jamais plus de $2,5 \%$ de la biomasse phytoplanctonique.

Chaque année, le genre Dinobryon avec $D$. bavaricum comme espèce dominante (surtout en été) présente un pic très court au début de la stratification (fig. 7B) ; toutefois en 1986, le maximum annuel beaucoup moins élevé qu'en 1987 se situe au début du printemps. Au sein de la biomasse phytoplanctonique, Dinobryon sp. représente au maximum 

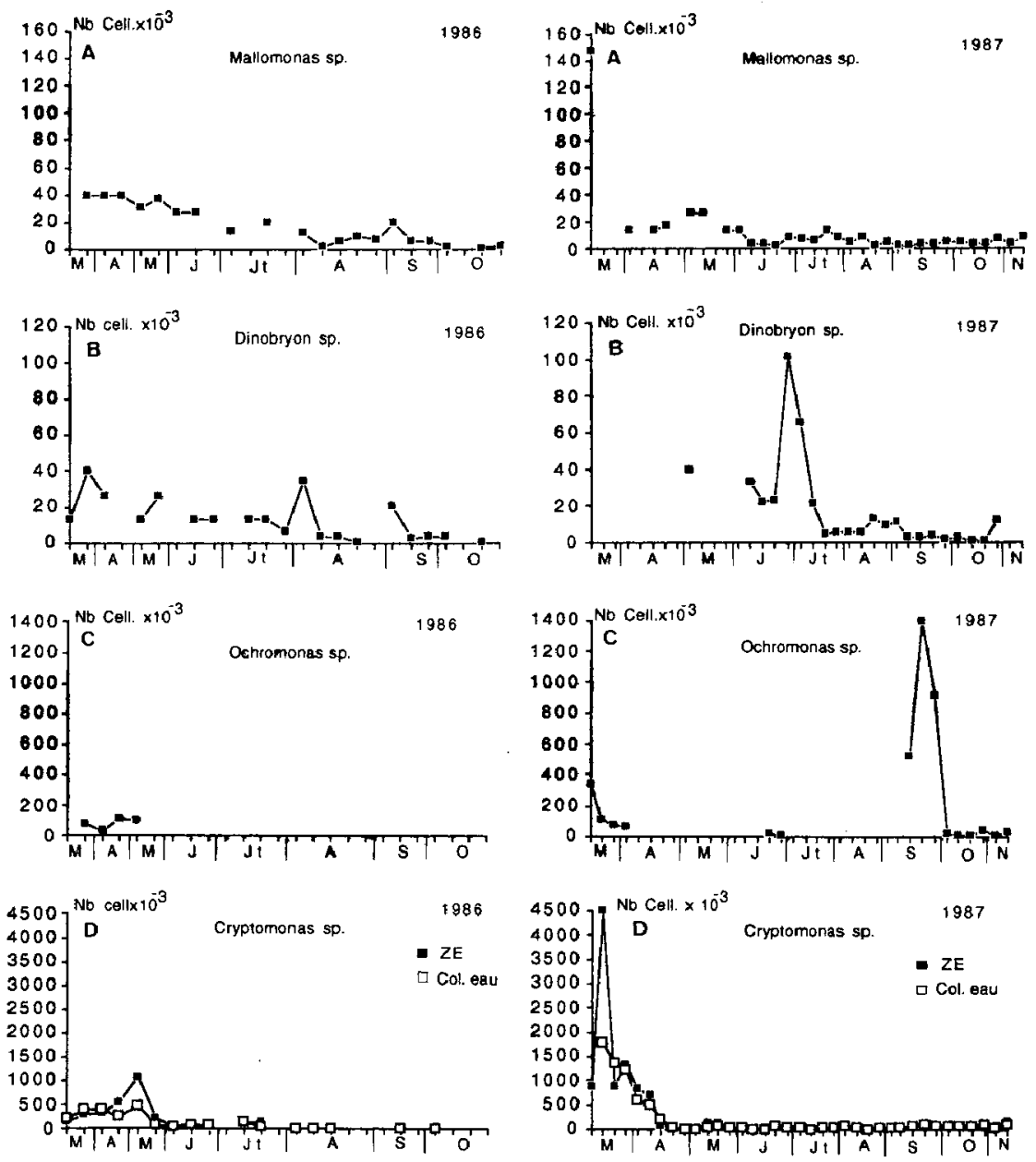

Fig. 7. Dynamique saisonnière (exprimée en nombre de cellules par litre) des genres : Mallomonas (A), Dinobryon (B), Ochromonas (C) et Cryptomonas (D). (Si non précisé sur le graphique, les résultats concernent toute la colonne d'eau explorée). 
$2,5 \%$ la première année et $6,3 \%$ la seconde. Le cycle annuel de développement confirme de nombreuses observations antérieures : périodes de croissance à diverses saisons (Dokulil \& Skolaut 1986), développement après la floraison vernale des diatomées (Hutchinson 1967, Rodhe et al. 1958) souvent juste après l'établissement de la stratification (Dokulil \& Skolaut 1991). A ce moment, dans le réservoir de Pareloup, le temps de doublement de la population, constituée de $D$. bavaricum, est de l'ordre de $2,2 \mathrm{j}$. en 1986 et de $3,2 \mathrm{j}$. en 1987 ; au sein de la colonne d'eau, en milieu de journée, Dinobryon sp. a alors tendance à se placer dans la partie profonde de la zone euphotique (entre $7 \mathrm{~m}$ et $10 \mathrm{~m}$, fig. 10 et 11 ). L'apparition et la disparition rapide de Dinobryon sp. peut sans doute s'expliquer par un taux de sédimentation important et surtout par l'impact de la prédation dû au zooplancton herbivore (Dokulil \& Skolaut 1991) ; dans le réservoir de Pareloup, le temps de doublement de la population est donc sans doute loin de traduire le taux de croissance réel. Par ailleurs, même si sa croissance est loin d'être entravée par le phosphore (Lehman 1976), le genre Dinobryon peut se manifester comme un compétiteur dominant avec de très faibles concentrations en phosphore (Sandgren 1988) ; dans le cas présent, cette condition n'est jamais d'actualité puisque les teneurs en phosphore ne sont jamais limitantes pour la croissance des algues (fig. $1 \mathrm{~B}$ et $1 \mathrm{C}$ ).

Aussi bien en 1986 qu'en 1987, le genre Ochromonas n'apparaît dans le phytoplancton qu'à de très courtes périodes (fig. 7 C) : printemps en 1986, printemps et début d'automne en 1987. Ce n'est qu'en septembre 1987 qu'il est d'un apport relativement important dans la biomasse phytoplanctonique (21 \%, fig. 6).

\subsubsection{Pyrrhophytes}

Les cryptophycées, sont représentées par le genre Cryptomonas sensu Bourrelly 1985 ; le maximum annuel (fig. $7 \mathrm{D}$ ) se situe à la fin de l'hiver-début du printemps. En 1987, pendant un court moment (10 mars), Cryptomonas sp. dépasse Asterionella formosa en densité cellulaire $\left(45.10^{5}\right.$ contre $8.10^{5}$ cell. $1^{-1}$ ), il représente alors $20 \%$ de la biomasse phytoplanctonique (fig. 6) ; pendant tout le reste de l'étude, ce pourcentage ne dépasse jamais $8 \%$.

Les dinoflagellés sont représentés essentiellement par les genres Peridinium et Gymnodinium,
Ceratium hirundinella (O.F. Müll.) Schrank n'apparaissant que sous forme d'individus isolés. Ces dinoflagellés présentent soit 2 maximums soit un seul (fig. $8 \mathrm{~A}$ et $8 \mathrm{~B}$ ), l'un des deux se situant toujours au printemps ; ils se manifestent de manière continue dans l'expression de la biomasse phytoplanctonique seulement pendant la stratification (fig. 6), principalement grâce au genre Gymnodinium. Le pourcentage qu'ils représentent alors n'a qu'une valeur relative dans la mesure où les valeurs de biomasse sont faibles, comme c'est d'ailleurs le cas pour les chrysophycées, les chlorophycées ou les cyanophycées.

\subsubsection{Raphidophycées}

Cette classe est représentée par une seule espèce, Gonyostomum semen (Ehr.) Diesing, dont l'évolution saisonnière a déjà été traitée par ailleurs (Lc Cohu et al. 1989). La présence de cette algue constitue une des originalités du réservoir de Pareloup. premier lac français où cette espèce a été observée en grande quantité.

En 1987, pendant la stratification, les deux pics de biomasse (fig. 3 et 4 ) sont dus à deux phases de croissance de Gonyostomum semen qui constitue alors environ $70 \%$ (fig. 6) de la biomasse phytoplanctonique ( 65 et $50 \%$ si l'on considère toute la colonne d'eau explorée). Dans les deux cas, le maximum de population se concentre au dessous de $5 \mathrm{~m}$ de profondeur et cela se répercute de manière spectaculaire sur les profils verticaux de la chlorophylle a (fig. 5). Gonyostomum semen est une espèce qui fuit les fortes intensités lumineuses puisque, au milieu de la journée, les maximums de population se placent dans une zone où les intensités lumineuses sont inférieures à $90 \mu \mathrm{E} \mathrm{m}^{2} \mathrm{~s}^{-1}$ alors qu'en surface elles sont supérieures à $600{ }_{\mu} \mathrm{E} \mathrm{m}^{2} \mathrm{~s}^{-1}$. Pen dant la période d'isothermie automnale, la population de Gonyostomum semen représente entre $40 \mathrm{et}$ $60 \%$ de la biomasse (fig. 6) avec une distribution verticale quasiment homogène sur toute la colonne d'eau explorée. La stratégie adaptative de Gonyostomum semen, dans la mesure où l'intensité lumineuse est faible, couvre donc des conditions très différentes (stratification et isothermic).

\subsubsection{Chlorophycées}

Par rapport à d'autres lacs, il faut noter l'absence d'espèces filamenteuses, type Ulothrix, Mougeotia etc... . En 1987, pendant une courte période du pic 
printanier (fig. 6, avril), des volvocales, Pandorina morum (Müller) Bory et Eudorina elegans Ehr., ont constitué jusqu'à $50 \%$ de la biomasse phytoplanctonique. Par ailleurs les chlorophycées sont essentiellement représentées par des chlorococcales: Dictyosphaerium pulchellum Wood, Crucigenia tetrapedia (Kirchn.) West et G. S. West et Crucigeniella apiculata (Lemm.) Kom. (syn. : Crucigenia apiculata (Lemm.) Schmidle), Sphaerocystis schroeteri Chod. . Leur développement principal se situe en été et en automne. Il faut noter que ce sont des espèces soit coenobiales soit arbustives plus ou moins adaptées à la flottaison mais présentant par ailleurs les caractères d'espèces à stratégie r. De plus, de par leur taille, elles sont facilement consommées par les prédateurs. Leur importance relative dans le peuplement (fig. 6) est liée, comme pour les groupes précédents, aux faibles valeurs de la biomasse phytoplanctonique.

\subsubsection{Cyanophycées}

Les cyanophycées apparaissent de manière significative pendant la période de stratification (fig. 6) ; leur présence peut être sporadique (1986) ou continue jusqu'au milieu de l'automne (1987). Gomphosphaeria naegeliana (Unger) Lemm., Anabaena spiroüdes Klebh. et Merismopedia tenuissima Lemm. en sont les éléments les plus représentatifs. D'une année sur l'autre, leur participation dans la constitution de la biomasse varie considérablement, de $20 \%$ en 1986 à $60 \%$ en 1987 (fig. 6) ; comme les chrysophycées, l'abondance relative des cyanophycées n'est élevée qu'aux périodes où les valeurs de la biomasse sont faibles. Cette relative importance est due au développement de Gomphosphaeria naegeliana (fig. $8 \mathrm{E}$ ) qui est l'espèce dominante en densité cellulaire pendant presque toute la période de stratification (fig. 12). Anabaena spiroides, seulement détectable en 1987, a son maximum en automne (fig. $8 \mathrm{D})$ mais pendant la stratification, elle est une composante non négligeable du peuplement en densité cellulaire (fig. 12). Merismopedia tenuissima, lors des deux années d'étude, a un cycle de développement très court au début de la période de brassage des eaux (fig. $8 \mathrm{C}$ ). Ces deux dernières espèces, importantes en densité cellulaire, ont un impact tout à fait mineur sur les valeurs de la biomasse.

\section{Conclusion}

Selon Sommer (1986), l'écart entre minimums et maximums annuels de biomasse au sein d'un même lac s'amplifie avec le degré de trophie : rapport de 1 à 10 pour les lacs oligotrophes, de 1 à 250 pour les lacs eutrophes. Avec des valeurs annuelles de la biomasse variant dans un rapport de 1 à 25-30, avec des concentrations en chlorophylle a peu élevées et enfin avec des teneurs en phosphore total de l'ordre de 14-25 $\mu$ g.1-1 (Sommer et al. 1986) à la fin de l'hiver, le réservoir de Pareloup peut se ranger dans la catégorie des lacs faiblement mésotrophes. Le cycle annuel de développement du phytoplancton a tendance à être de type bimodal avec une poussée printanière toujours spectaculaire et une poussée automnale beaucoup moins accentuèe se manifestant surtout par une augmentation d'une part des concentrations de la chlorophylle a (fig. 4) et d'autre part de la densité cellulaire (fig. 3).

Dans le modèle de succession phytoplanctonique, modèle P.E.G., proposé par Sommer et al. (1986), l'évolution saisonnière des peuplements phytoplanctoniques, du printemps à l'automne, est déterminée par des facteurs biologiques (compétition, broutage) et chimiques (déficience en éléments nutritifs) ; les facteurs physiques, lumière, température et brassage, ne deviennent prépondérants qu'en automne et en hiver. Dans le réservoir de Pareloup, les quatre premières phases du modèle $P$.E.G. se réduisent à une seule, dominée par Asterionella formosa. La présence d'algues susceptibles d'être consommées par le zooplancton tel le genre Cryptomonas (phase 1 du modèle P.E.G.) qui atteint pourtant son maximum au printemps (fig. 7 D) est effacée. Asterionella formosa se confirme comme étant une espèce à faible exigence vis à vis de la lumière, comme étant une excellente compétitrice lorsque le rapport $\mathrm{Si} / \mathrm{Int}$. lum. est élevé (Sommer 1989) et lorsqu'elle est confrontée à des variations d'intensité lumineuse (Reynolds \& Reynolds 1985, Sommer 1987). De même un fort rapport Si/Pt favorise sa croissance (Sommer 1989 b) ; dans le cas présent, les pertes par sédimentation commencent à l'emporter sur son taux de renouvellement lorsque le rapport $\mathrm{Si} / \mathrm{Pt}$ tend vers ses valeurs les plus faibles (\#15 fig. $1 \mathrm{E}$ ) ; la silice devient alors limitante (fig. 2 A) mais il n'est pas exclu que, pendant un court moment $(23 / 5 / 86$; 13/5/87), le PRS le soit aussi (les valeurs de ce dernier recommencent d'ailleurs à augmenter lorsque le processus d'intense sédimentation est enclenché). Le rapide déclin d'Asterionella formosa peut être également déclenché en partie, comme nous l'avons signalé précédemment, par le déficit physiologique, 

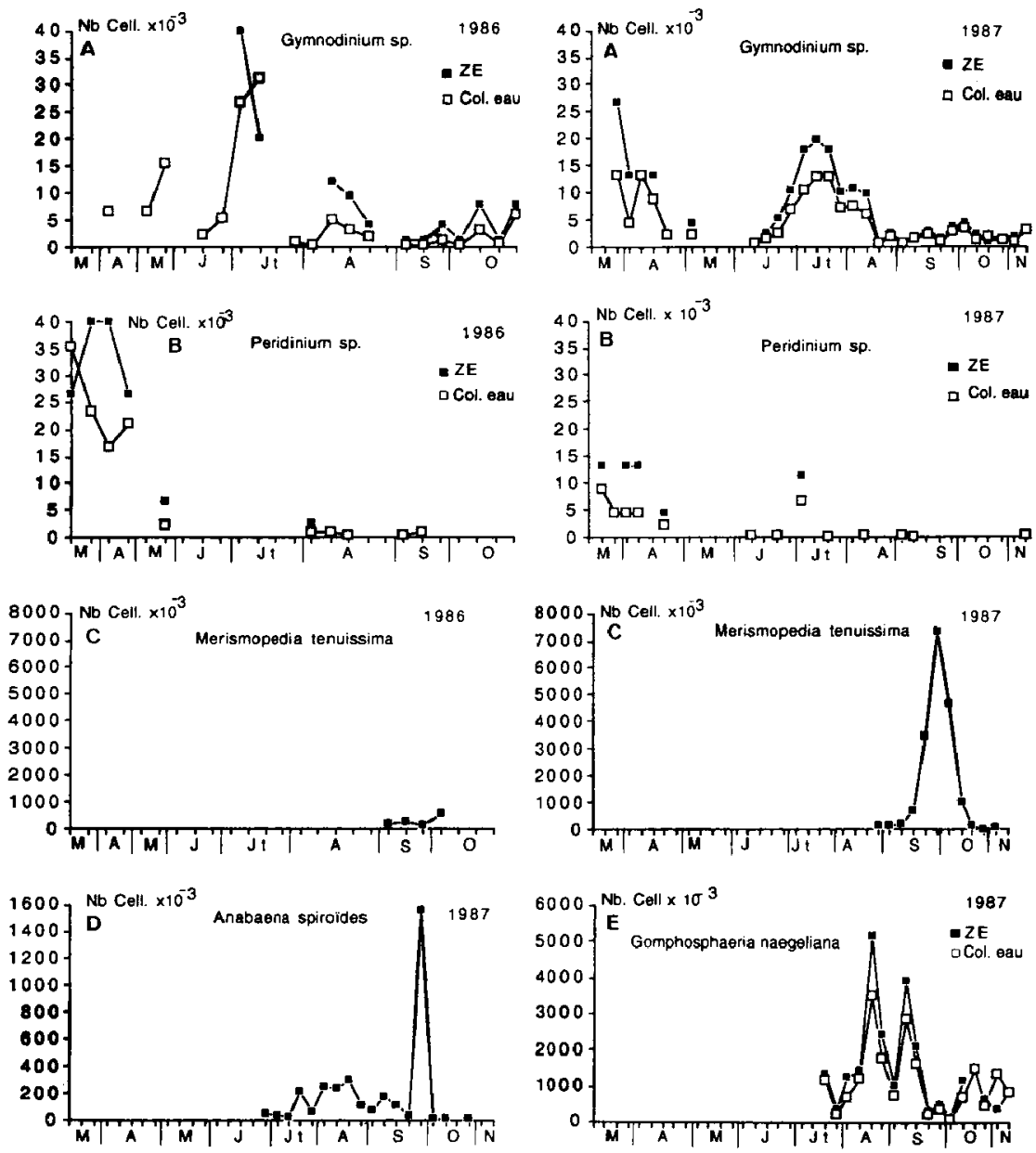

Fig. 8. Dynamique saisonnière (exprimée en nombre de cellules par litre) de Gymnodinium sp. (A), Peridinium sp. (B), Merismopedia tenuissima (C), Anabaena spiroïdes (D) et Gomphosphaeria naegeliana (E). (Si non précisé sur le graphique, les résultats concernent toute la colonne d'eau explorée). 
provoqué par les concentrations cellulaires minimales en chlorophylle a au moment du maximum de cette espèce.

Le lac de Pareloup appartient donc à cette catégorie de lac de profondeur moyenne où la floraison print anière est dominée par les fragilariacées (Sommer 1989 b) ; cependant, dans le cas présent, le processus de sédimentation est achevé avant la mise en place de la stratification thermique ; la clarification des eaux qui en résulte est donc essentiellement initiée par une carence en un élément nutritif $\left(\mathrm{SiO}_{2}\right)$ et non par le broutage comme dans le modèle P.E.G. (Sommer et al.1986). Cet approfondissement de la zone euphotique va se prolonger pendant tout l'été. Début de la phase claire et établissement de la stratifiction correspondent au minimum annuel de la biomasse mais marquent aussi un remaniement profond de la structure du peuplement avec une augmentation de la diversité (fig. 12). L'établissement de la stratification thermique correspondrait ici à la fin de la succession autogénique sensu Reynolds 1984. Pendant toute la phase de stratification, la croissance algale n'est limitée ni par l'azote (fig. 1 A et 2 B) ni par le phosphore (fig. 1 B et 1 C) rapidement recyclé (Thébault \& Salençon, sous presse) : maximum de production primaire (Capblancq et al. 1988) et faibles valeurs globales de la biomasse (fig. 3 et 4) caractérisent toute cette période ; le développement algal est régulé par la prédation (Thébault $\&$ Salençon, sous presse). On a affaire à une biocénose algale instable dont les taxons dominants (en densité cellulaire ou en biomasse) peuvent changer rapidement (fig. 9 à 12). Une autre caractéristique de cette biocénose algale est une grande hétérogénéité verticale de la densité cellulaire et des concentrations en chlorophylle a. Ces fluctuations (fig. 5, 9 et 12) sont dues d'une part à la croissance momentanée de taxons type Dinobryon ou d'espèces de petite taille à rapport $\mathrm{S} / \mathrm{V}$ élevé (type Dictyosphaerium pulchellum) et d'autre part au développement tout aussi transitoire de Gonyostomum semen (Le Cohu et al. 1989). Les algues, sauf Anabaena spiroides, ont tendance à se placer dans la partie inférieure de la zone euphotique (fig. 9 à 12), ce qui traduit sans doute la formation de " niches écologiques » spatiales déjà mentionnées par Reynolds (1984). Seules les cyanophycées, Gomphosphaeria naegeliana et Anabaena spiroildes, à partir de fin juillet, ont une présence continue et dominent en densité cellulaire (fig. 12) ; (rappelons que pour le calcul des biovolumes ce sont les colonies de Gomphosphaeria qui ont été prises en compte et cela explique, en grande partie, la part relativement importante prise par cette espèce dans la composition de la biomasse fig. 6).

Le brassage automnal des eaux est marqué par le développement, relativement important en densité cellulaire, d'espèces de petite taille (Ochromonas, Merismopedia tenuissima), par la part conséquente prise par Gonyostomum semen dans les valeurs de biomasse, par le retour des diatomées printanières ou même parfois d'espèces estivales (Dictyosphaerium pulchellum).

Le modèle P.E.G. (Sommer et al. 1986) peut difficilement s'appliquer au réservoir de Pareloup qui s'en distingue par plusieurs particularités qui nous ont amenés à proposer une nouvelle approche de la succession phytoplanctonique.

1) La phase claire est induite par la sédimentation des diatomées et se prolonge pendant tout l'été.

2) L'établissement de la stratification est un facteur fondamental dans la mesure où se crée un milieu carencé en silice et avec un rapport $\mathrm{Si} / \mathrm{Pt}$ faible ; le stade 11 du modèle P.E.G. (développement des grandes diatomées) ne peut se réaliser ; les espèces à rapport $\mathrm{S} / \mathrm{V}$ élevé sont favorisées mais leur développement est contrôlé par la prédation. On a alors des valeurs de biomasse qui ne rendent sans doute pas compte de l'état trophique réel du lac.

3) La présence de Gonyostomum semen se traduisant de façon importante dans la biomasse donne un caractère original à la retenue de Pareloup (Le Cohu et al. 1989). Cette espèce présente certaines des particularités d'une espèce de stratégie $\mathrm{K}$ : grande taille, faible rapport $\mathrm{P} / \mathrm{B}$ (Capblancq et al. 1988) mais, par ailleurs, elle ne résiste pas à la prédation et s'adapte très bien à la turbulence. Il faut d'ailleurs remarquer qu'une espèce comme Ceratium hirundinella, reconnue typiquement de stratégie $\mathrm{K}$ (Reynolds 1984) et présentée comme ayant son maximum de développement pendant la période de stratification (Sommer et al. 1986), peut avoir un comportement totalement différent : par exemple dans le lac Hassan 1 $1^{\mathrm{er}}$, Maroc (Hasnaoui, comm. pers.) on observe son maximum à la fin du printemps et en automne, en périodes d'isothermie.

4) Dans le réservoir de Pareloup, si l'on souscrit au concept de succession phytoplanctonique proposé par Reynolds (1984) ou par d'autres (Amblard 

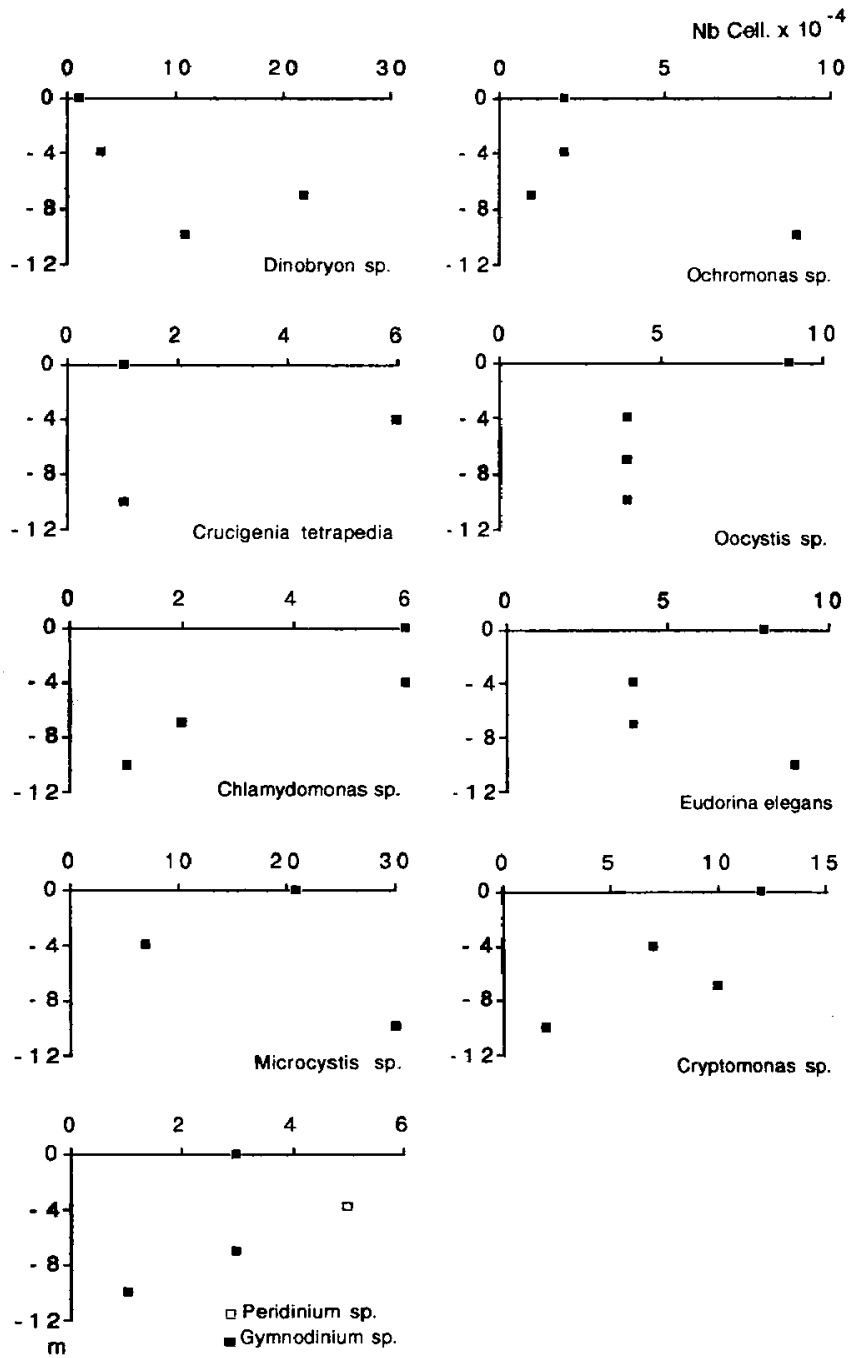

Fig. 9. Distribution verticale de quelques genres ou espèces le $9 / 7 / 87$ dans la zone euphotique (exprimée en nombre de cellules par litre). 

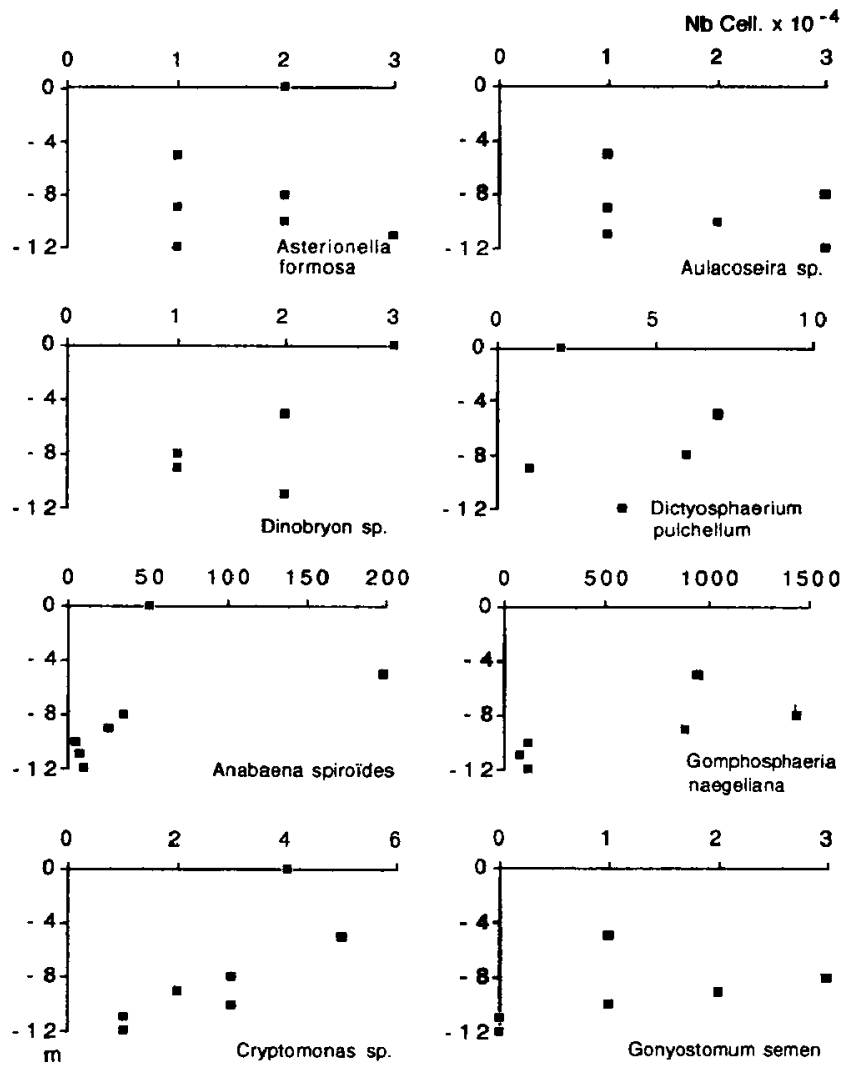

Fig. 10. Distribution de quelques genres ou espèces le $18 / 8 / 87$ dans la zone euphotique (exprimée en nombre de cellules par litre). 

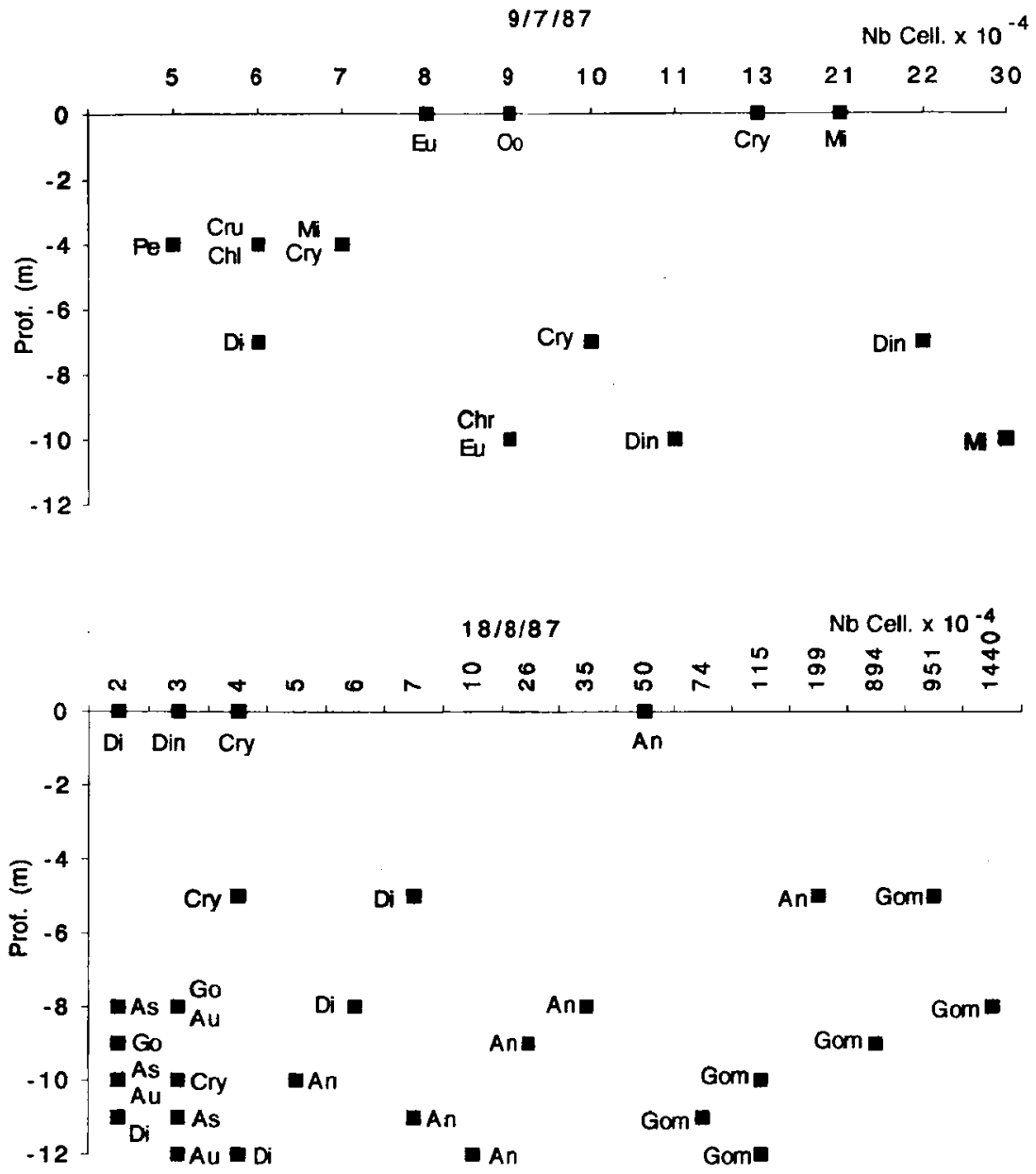

Fig. 11. Répartition verticale de la densité cellulaire des genres dominants dans la zone euphotique le $9 / 7 / 87$ (A) et le 18/8/87 (B.) (exprimée en nombre de cellules par litre). An : Amabaena, As : Asterionella, Au : Aulacoseira, Chl : Chlamydomonas, Chr : Chrysophycée, Cru : Crucigenia, Cry : Cryptomonas, Di : Dictyosphaerium, Din : Dinobryon, Eu: Eudorina, Go : Gonyostomum, Gom : Gomphosphaeria, Mi : Microcystis, Oo : Oocystis, Pe : Peridinium. 


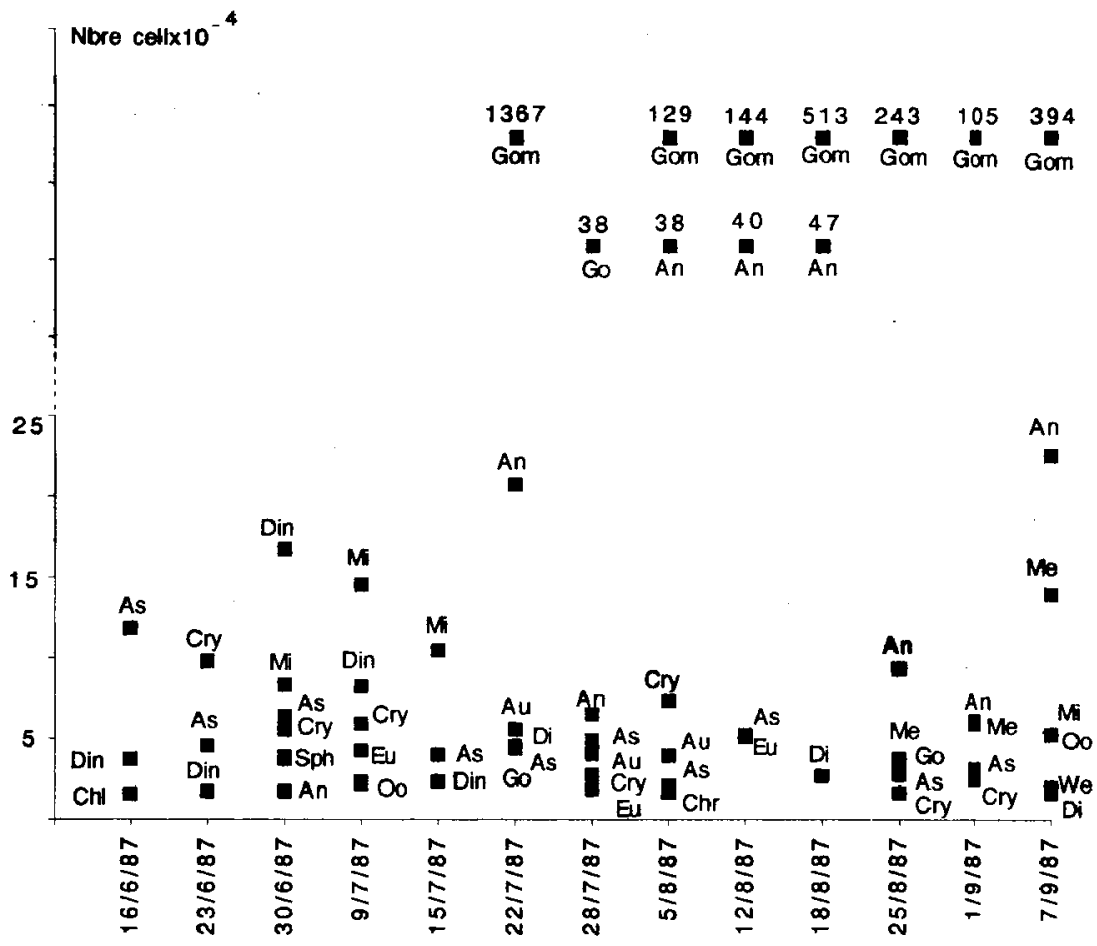

Fig. 12. Evolution de la densité cellulaire des genres dominants dans la zone euphotique pendant la période de stratification thermique du lac (exprimée en nombre de cellules par litte). An : Anabaena, As : Asterionella, Au : Aulacoseira, Chr : Chrysophycé, Cru : Crucigenia, Cry : Cryptomonas, Di : Dictyosphaerium, Din : Dinobryon, Eu : Eudorina, Go : Gonyostomum, Me : Merismopedia, Mi : Microcystis, Oo : Oocystis, Pe : Peridinium, Sph : Sphaerocystis, We : Westella.

1987), l'établissement de la stratification provoque la fin de la succession autogénique proprement dite avec un retour vers des stades plus juvéniles au sein du biotope. Cette succession autogénique ser ait donc interrompue par une perturbation allogénique (stratification) qui change la direction de la succession avec apparition d'un milieu seulement carencé en silice ; or, dans le réservoir de Pareloup, l'établissement de la stratification thermique n'est pas un événement aléatoire, imprévisible, c'est au contraire un élément fondamental du fonctionnement du lac ; la succession devient alors de type cyclique, telle que la définit Ramade (1984) : démarrage de la succession au départ de la stratification et stade ultime au printemps avec le maximum de biomasse. Dans la succession phytoplanctonique, la lumière est considérée comme une ressource au même titre que les autres facteurs nécessaires à la croissance des algues. 


\section{Travaux cités}

Amblard (C.). 1987. - Les successions phytoplanctoniques en milieu lacustre. Ann. Biol, XXI : 1-34.

Amblard (C.). 1988. - Seasonal succession and stategies of phytoplankton development in two lakes of different trophic states. J. Plankton Res., 10, 6 : 1189-1208.

Amblard (C.) \& Bourdier (G.). 1990. - The spring bloom of the diatom Melosira italica subsp. subarctica in lake Pavin : biochemical, energetic and metabolic aspects during sedimentation. J. Plankton Res., 12 (3) : 645-690.

Bourrelly (P.). 1985. - Les algues d'eau douce, T. III: les algues bleues et rouges. Soc. Nouv. Ed. Boubee, Paris, $606 \mathrm{p}$.

Capblanca (J.), Thébault (J.M.) \& Jrad (A.). 1988. - Relations entre la lumière et la photosynthèse du phytoplancton dans un réservoir mésotrophe (Pareloup) : variations saisonnières des paramètres. Annls Limnol., $24: 39-48$.

Devaux (J.). 1973. - Contribution à l'étude des populations phytoplanctoniques du lac Tazenat (Puy de Dôme). Ann. Star. Biol. Besse-en-Chandesse, 7 : 1-101.

Devaux (J.). 1977. - Dynamique des populations phytoplanctoniques dans deux lacs du Massif Central. Ann. Stat. Biol. Besse-en-Chandesse, 10 Suppl. : $185 \mathrm{p}$.

Dokulil (M.T.) \& Skolaut (C.). 1986. - Succession of phytoplankton in deep stratifying lake : Mondsee, Austria. Hydrobiologia, 138: 9.24.

Dokulil (M.T.) \& Skolaut (C.). 1991. - Aspects of phytoplankton seasonal succession in Mondsee, Austria, with particular reference to the ecology of Dinobryon Ehrenb. Verh. Internat. Verein. Limnol., 24 : 968-973.

Falkowski (G.P.). 1984. - Physiological responses of phytoplankton to natural light regimes. J. Plankton Res., 6 : 295-307.

Hoepffner (N.). 1984. - Stratégies d'adaptation photosynthetique chez les diatomées de l'océan Ant arctique : variations du nombre et de la taille des unités photosynthétiques. $J$. Plankton Res., 6 (5): 881-895.

Hutchinson (G.E.). 1967. - A treatise of Limnology, vol. II. Willey, New York. 1115 p.

Jrad (A.). 1989. - Phytoplancton el production primaire dans le lac de Pareloup (Aveyron). Thèse de doctorat 3ème cycle, Université Toulouse III : $166 \mathrm{p}$.

Labroue (L.). 1990 . - Echanges sédiments - eau dans le lac de Pareloup. Colloque Hydrócologie EDF Paris : 6-7.

Lavandier (P.). 1990. - Dynamics of bacterioplankton in a mesotrophic French reservoir (Pareloup). Hydrobiologia, $207: 79-86$

Le Cohu (R.). 1973. - Utilisation d'enceintes en milieu naturel pour l'étude de l'influence des nitrates sur les populations algales. C.R. Acad. Sci., $276: 1565-1568$

Le Cohu (R.), Guitard (J.), Comoy (N.) \& Brabet (J.). 1989. Gonyostomum semen (Raphidophycées), nuisance potentielle des grands réservoirs français ? L'exemple du lac de Pareloup, Arch. Hydrobiol., 117 (2) : 225-236.

Lehman (J.T.). 1976. - Ecological and nutritional studies on Dinobryon Ehrenb. : Seasonal periodicity and the phosphat toxicity problem. Limnol. Oceanogr., 21 : 646-658.
Le Jolivet (C.). 1988. - Contribution à l'étude du recrutement des poissons du réservoir de Pareloup (Aveyron). Thèse de doctorat, I.N.P. Toulouse : $288 \mathrm{p}$.

Lund (J.W.G.), Kipling (C.) \& Le Cren (E.D.). 1958. - The inverted microscope method of estimating algal numbers and the statistical bases of estimations by counting. Hydrobiologia, $11: 143-170$.

Lund (J.W.G.). 1964. - Primary production and periodicity of phytoplankton. Verh. Int. Ver. Limnol., $15: 37-56$.

Marker (A.F.H.), Nush (E.A.) \& Riemann (B.). 1980. - The measurements of photosynthetic pigments in freshwater and standardisation of methods : conclusions and considerations. Ergeb_Limnol., $14: 91-106$.

Motomizu (S.), Wakimoto (T.) \& Tôei (K.). 1983. Spectrophotometric determination of phosphate in river waters with molybdate and malachite green. Analyst., 108 : $36 !-367$.

Ramade (F.). 1984, - Elements d'écologie. Mc Graw Hill, Paris : 403 p.

Reynolds (C.S.). 1984. - The ecology of freshwater phytoplankton. Cambridge Univ. Press, Cambridge, $384 \mathrm{p}$.

Reynolds (C.S.) \& Reynolds (J.B.). 1985. - The atypical seasonality of phytoplankt on in Cros Mere, $1972:$ an independant test of the hypothesis that variability in the physical environment regulates community dynamics and structure. British Phycological Journal, $20: 227-242$.

Rhodes (T.E.) \& Le Cohu (R.), 1987. - Sédiments et paléolimnologie d'un réservoir : le lac de Pareloup (Aveyron, France). Premiers résultats. Amnls Limnol., $23: 3-7$.

Rodhe (W.), Vollenweider (R.A.) \& Nauwerk (A.). 1958. - The primary production and standing crop of phytoplankton. In : Buzzati \& Travers A.A. (Ed.) : Perceptives in marine bio$\log y$ : 294-322. Berkeley, Los Angeles.

Sandgren (C.D.). 1988. - The ecology of chrysophyte flagellates : their growth and perennation strategies as freshwater phytoptankton. In : Sandgren C.D. (Ed.) : Growth and reproductive strategies of freshwater phytoplankton : 9-106. Cambridge Univ, Press, Cambridge.

Sommer (U.). 1986. - The periodicity of phytoplankton in Lake Constance (Bodensce) in comparison to other deep lakes of central Europe. Hydrobiologit, $138=1-7$.

Sommer (U.). 1987. - Factors controlling the seasonal varia. tion in phytoplank ton species composition. A case study for a deep nutrient rich lake. Progress in Phycological Research, 5: $123-178$.

Sommer (U.) (Ed.). 1989a, - Plankton ecology. Succession in Plankson community. Springer-Verlag, Berlin ; $360 \mathrm{p}$.

Sommer (U.). 1989b. - The role of competition for resources in phytoplankion succession. In : Sommer (U.) (Ed.) : Plankron ecology - Springer Vẹrlag, Bęrlin : \$7-106.

Sommer (U.), Gliwicz (Z.M.), Lampert (W.) \& Duncan (A.). 1986. - The P.E.G. model of seasonal succession of planktonic events in freshwaters. Archiv. fiur Hydrobiol. 433-471.

Thébault (J.M.) \& Salençon (M.J.) (sous presse). - Simulation model of a mesotrophic reservoir (Lac de Pareloup, France) : Biological model. Ecol. Modelling. 